\title{
Down-ramp injection and independently controlled acceleration of electrons in a tailored laser wakefield accelerator
}

\author{
M. Hansson, ${ }^{1, *}$ B. Aurand, ${ }^{1}$ X. Davoine, ${ }^{2}$ H. Ekerfelt, ${ }^{1}$ K. Svensson, ${ }^{1}$ \\ A. Persson, ${ }^{1}$ C.-G. Wahlström, ${ }^{1}$ and O. Lundh ${ }^{1, \dagger}$ \\ ${ }^{1}$ Department of Physics, Lund University, P.O. Box 118, S-22100 Lund, Sweden \\ ${ }^{2}$ CEA, DAM, DIF, Bruyères-le-Châtel, 91297 Arpajon, France
}

(Received 23 March 2015; published 8 July 2015)

\begin{abstract}
We report on a study on controlled injection of electrons into the accelerating phase of a plasma wakefield accelerator by tailoring the target density distribution using two independent sources of gas. The tailored density distribution is achieved experimentally by inserting a narrow nozzle, with an orifice diameter of only $400 \mu \mathrm{m}$, into a jet of gas supplied from a $2 \mathrm{~mm}$ diameter nozzle. The combination of these two nozzles is used to create two regions of different density connected by a density gradient. Using this setup we show independent control of the charge and energy distribution of the bunches of accelerated electron as well as decreased shot-to-shot fluctuations in these quantities compared to self-injection in a single gas jet. Although the energy spectra are broad after injection, simulations show that further acceleration acts to compress the energy distribution and to yield peaked energy spectra.
\end{abstract}

DOI: 10.1103/PhysRevSTAB.18.071303

PACS numbers: 41.75.Jv, 52.35.-g, 52.38.-r, 52.50.Jm

Benefiting from the high electric fields that can be sustained in a plasma wave, laser wakefield accelerators [1] appear promising as compact sources of highly relativistic electrons and X-rays. Quasi-monoenergetic bunches of highly relativistic electrons were first observed in 2004 [2-4], by self-injection through wave-breaking. Since then, much effort has been made on controlling the injection of electrons into the accelerating plasma structure. Different mechanisms for injection, such as injection by colliding laser pulses [5-7] and ionization [8-11] and injection in density down-ramps [12-16] etc., have been proposed and studied both theoretically and experimentally.

The mechanism of density down-ramp injection is typically divided into two regimes; short density ramps (of the order of the plasma wavelength, $\lambda_{p}$ ) and long density ramps $\left(>\lambda_{p}\right)$. Short density ramps have been produced experimentally, for example by optical plasma formation and expansion [17] and by shock waves [15,18] in gas jets. Due to the well-localized injection point, electron bunches with peaked energy spectra can be generated. In longer density ramps $[14,16]$, injections occur over a longer distance, and thus initially give broad energy spectra. However, after further acceleration of the electrons, the energy spectra can become peaked.

The mechanism of density down-ramp injection relies on breaking of the plasma density wave that follows a laser

\footnotetext{
*martin.hansson@fysik.lth.se

olle.lundh@fysik.lth.se
}

Published by the American Physical Society under the terms of the Creative Commons Attribution 3.0 License. Further distribution of this work must maintain attribution to the author(s) and the published article's title, journal citation, and DOI. pulse. This occurs when the electrons that constitute the plasma wave approaches and exceeds the phase velocity of the wave. For laser wakefield accelerators based on selfinjection this is achieved by driving the plasma density oscillations to such high amplitude that wave-breaking occurs. In contrast, density down-ramp injection exploits the gradually increasing plasma wavelength in the ramp. Behind the driving laser pulse, this results in a decreased phase velocity of the plasma density wave and can thus be used to reach the conditions for wave-breaking.

In this article, we present a study, experimentally and numerically, on controlled injection of electrons into the accelerating field of a laser wakefield accelerator, based on long density down-ramps, and the subsequent acceleration of the injected electrons in the following low density plasma. The aim is to improve our understanding of the physics behind both injection in density down-ramps and the subsequent acceleration.

Controlled injection is achieved in this experiment using two separate nozzles to supply the gas in the interaction region. In contrast to the work presented in Ref. [16], where ionization-induced injection is employed in combination with a density down-ramp, the electrons are injected in this experiment solely by density down-ramp injection. Furthermore, our experimental setup allows for continuous variation of the length of the plasma after the injection point, as compared to Refs. $[14,16]$. We show that this density distribution can be used to separately control the amount of charge and the electron kinetic energy in the bunches of accelerated electrons. The shot-to-shot fluctuations, in total charge and energy distribution, achieved using this setup are significantly smaller compared to the beams accelerated in the self-injection scheme in a single 
gas jet. The conclusions presented in this article are based on experiments, performed using a multi-terawatt laser at the Lund Laser Centre, and supported by particle-in-cell (PIC) simulations using the code CALDER-CIRC [19].

The laser pulses, each containing $650 \mathrm{~mJ}$ of energy and with a duration (FWHM) of $40 \mathrm{fs,} \mathrm{are} \mathrm{focused} \mathrm{to} \mathrm{an} \mathrm{almost}$ circular spot with $19 \mu \mathrm{m}$ diameter (FWHM), using an $f=0.765 \mathrm{~m}$ off-axis parabolic mirror. The peak intensity of the laser pulses, when focused in vacuum, is determined to $3.7 \times 10^{18} \mathrm{~W} / \mathrm{cm}^{2}$, corresponding to a normalized vector potential of 1.3 .

Two separate nozzles are used to provide the desired density distribution of hydrogen gas in the interaction region, as illustrated in Fig. 1(a), and is ionized by the leading edge of each laser pulse. The main part of the gas is supplied by a nozzle with an exit diameter of $2 \mathrm{~mm}$, with its orifice located $1 \mathrm{~mm}$ from the optical axis. This nozzle provides an almost cylindrically symmetric jet of gas toward the optical axis, and is typically positioned such that the laser pulse is focused on the front edge of the density distribution. Additionally, a narrow metallic tube, with an orifice diameter of $400 \mu \mathrm{m}$, is inserted into the jet, perpendicular to both the optical axis and the direction of the main jet. Gas is supplied through this tube to provide an additional, localized, contribution to the density in the interaction region with the laser pulse.

The total neutral density distribution, along the optical axis, of the gas provided from these two nozzles is characterized off-line by measuring, using a wavefront sensor, the additional optical path length introduced by the gas in an optical probe beam [20]. The optical path length introduced by the gas provided from the $2 \mathrm{~mm}$ nozzle is first measured and the density distribution is calculated assuming circular symmetry. The narrow tube is inserted into the flow from the $2 \mathrm{~mm}$ nozzle and the wavefront is again measured, first without any gas supplied from the narrow tube. By comparing the wavefront with and without the narrow tube inserted in the flow we conclude that the gas distribution is essentially unaffected by inserting this tube. Finally, the difference in optical path length is measured with gas supplied simultaneously from the main nozzle and from the narrow tube, as shown in Fig. 1(b). This allows the contribution from the narrow tube to the total gas density to be determined assuming circular symmetry close to the orifice, and the final total density profile, shown in Fig. 1(c), to be calculated.

The total density distribution along the optical axis contains a peak and a plateau joined together by a gradient. As will be shown, under suitable chosen conditions, density down-ramp injection of electrons into the accelerating phase of a laser plasma wakefield occurs in this gradient and the electrons are subsequently accelerated in the remaining plasma.

The backing pressures supplied independently to each nozzle are used to control the density in the peak and the plateau. The density profile from the $2 \mathrm{~mm}$ gas nozzle is approximately flat over $0.7 \mathrm{~mm}$ which corresponds to the maximum plateau length. The density in the plateau is used to control the plasma wavelength in this region and is also used to tune the strength of the accelerating field. Furthermore, the two nozzles are separately mounted on 3-axis translation stages which allow full control of the position of the two density distributions both relative to each other and relative to the laser focus. By moving the $2 \mathrm{~mm}$ nozzle along the optical axis, while keeping the narrow nozzle fixed, the length of the density plateau is varied. This degree of freedom provides a mean to perform studies of the acceleration independently of the injection of electrons.

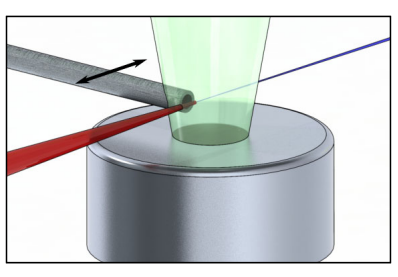

(a)

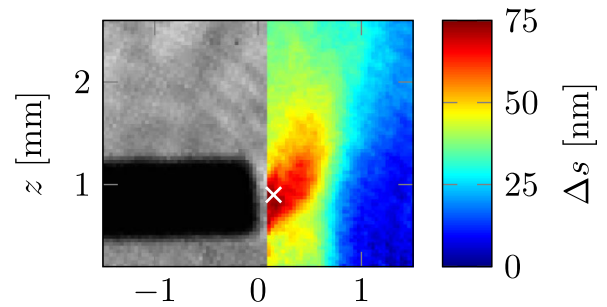

(b) $y[\mathrm{~mm}]$

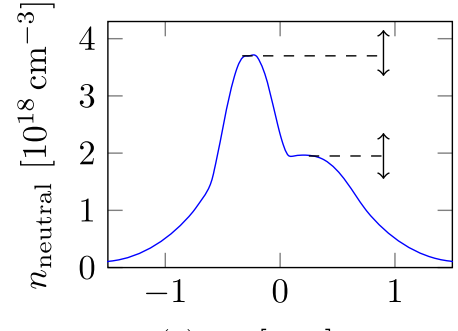

(c) $x[\mathrm{~mm}]$

FIG. 1. A schematic illustration of the experimental setup is shown in (a). The laser pulses (red) are focused on the front edge of the gas jet provided from a $2 \mathrm{~mm}$ nozzle with its orifice located $1 \mathrm{~mm}$ from the optical axis. A narrow tube is inserted into the jet, with its orifice $0.2 \mathrm{~mm}$ from the optical axis, and provides locally an additional amount of gas. The electrons (blue) accelerated in the interaction propagate along the optical axis. Measurements, using a wavefront sensor, of the additional optical path length introduced by the gas in an optical probe beam allows the neutral gas density profile to be determined. In (b) the additional path length $(\Delta s)$ introduced by the gas, supplied from both nozzles simultaneously, is shown in the color scale in the part not obstructed by the narrow tube. The 2 mm nozzle is located just below the edge of the image and supplies a flow of gas along the vertical ( $z$ ) axis. The shadow of the narrow tube marks its position in the left part of the figure. The optical axis of the main laser beam is perpendicular to the plane of the figure and its position in the plane is marked as a white cross. The typical neutral gas density $\left(n_{\text {neutral }}\right)$ distribution along the optical $(x)$ axis used in this experiment is shown in (c). The tube can be moved along the optical axis to change the position of the density peak and thus also the density down-ramp. Furthermore, the density in the peak and plateau can be varied independently. 
Measurements of the density distributions show that the gradient between the two regions is approximately $230 \mu \mathrm{m}$ long and is unaffected by changing the backing pressure within the range used in this experiment. Thus, the density down-ramp becomes sharper as the backing pressure to the narrow tube is increased, which allowed for studies to be performed of the dependence of the number of injected electrons on the gradient.

The electrons accelerated in the plasma are observed by letting them impact on a scintillating screen (KODAK LANEX Regular), imaged onto a 16-bit CCD-camera (PrinCETON PhotonMAX 1024). The amount of charge impacting on the scintillating screen is determined using published calibration factors for the screen [21] and by calibration of the response of the CCD-camera through the imaging optics. Furthermore, a $10 \mathrm{~cm}$ long dipole magnet with a peak field strength of $0.7 \mathrm{~T}$ can be inserted in the electron beam to disperse the electrons according to energy before impacting on the scintillating screen. This allows for the energy spectrum, above a cutoff energy of $40 \mathrm{MeV}$, of the electron beams to be determined. The electron energy dispersion on the scintillating screen was calibrated by numerically tracing electrons of different energies through the dipole magnetic field, according to the experimental geometry.

Electrons were first injected and accelerated in a target where gas was supplied only from the $2 \mathrm{~mm}$ gas nozzle. The threshold in electron number density in the plateau for required self-injection was found to be approximately $11 \times 10^{18} \mathrm{~cm}^{-3}$. The observed beams of electrons had the typical characteristics of self-injection in gas jets [22,23], with limited reproducibility and a bunch charge of the order of $30 \mathrm{pC}$ with a standard deviation higher than $50 \%$.

The electron number density provided from the $2 \mathrm{~mm}$ nozzle was lowered well below threshold for injection (to $3 \times 10^{18} \mathrm{~cm}^{-3}$ ). When adding gas also from the narrow tube, beams of accelerated electrons were observed [see Fig. 2(a)] for every laser pulse sent onto the target. The bunches of accelerated electrons injected using this composite gas target contain only of the order of $1 \mathrm{pC}$ and their spectra typically contain a broad peak [see Fig. 2(b)]. Furthermore, the shot-to-shot stability in charge and energy of the electron beams, with standard deviations $13 \%$ and $5 \%$, respectively, is far better than the stability of the beams injected through the self-injection mechanism in a single gas jet. This indicates that the local increase of gas in the interaction region facilitates the injection of electrons into the accelerating wakefield, and the reproducibility suggests that the mechanism is different from the self-injection observed when only supplying gas from one nozzle.

The kinetic energy of the accelerated electrons could be controlled by varying the remaining plasma length after the density down-ramp. This was done by moving the $2 \mathrm{~mm}$ gas nozzle, while keeping the position of the down-ramp

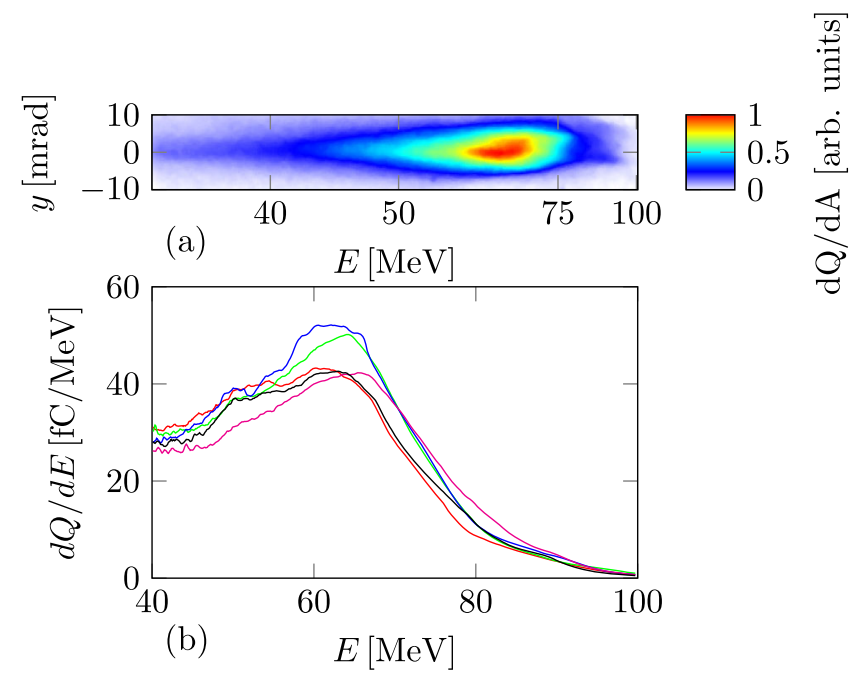

FIG. 2. Typical image of the dispersed electrons impacting on the scintillating screen (a) in a color map representing amount of charge per area. The total amount of charge is approximately $1.5 \mathrm{pC}$ and the beams have a divergence of $10 \mathrm{mrad}$. Calculated energy spectra of electrons accelerated in five consecutive shots (b). The energy spectra of the electrons accelerated using this target typically contains a broad peak at an energy that is tunable from 50 to $80 \mathrm{MeV}$. The shot-to-shot fluctuations in charge and average energy achieved using this setup are significantly better compared to self-injection.

fixed with respect to the laser focus in vacuum. The resulting dependence of the peak electron energy on the length of the remaining plasma is shown in Fig. 3 for two different densities in the plateau. The result shows that a longer plasma, after the density down-ramp, provides higher energy of the electrons. This corresponds well with the estimated dephasing length [24] $L_{d} \approx 3 \mathrm{~mm}$, i.e., the maximum length an injected electron can stay in the accelerating phase of the wakefield, which is much longer than the plateau.

Assuming that the movement of the $2 \mathrm{~mm}$ gas nozzle has minor effects on the position of injection, the average accelerating electric field is estimated by fitting a line to each series of data. This gives a value of $37 \mathrm{MV} / \mathrm{mm}$ at a density of $2.6 \times 10^{18} \mathrm{~cm}^{-3}$ in the plateau and $50 \mathrm{MV} / \mathrm{mm}$ at a density of $3.25 \times 10^{18} \mathrm{~cm}^{-3}$.

These accelerating electric fields are quite low compared to most other studies of laser wakefield acceleration using similar laser parameters [15]. This can be explained by two parts; first, the electron number density in the plateau is relatively low compared to studies in which the accelerator is operated close to the threshold for self-injection. This leads to a lower peak electric field in the accelerating region in our experiments. Second, as the electrons are injected when the plasma wake is growing longitudinally behind the laser pulse, in a long gradient, the electrons will be distributed longitudinally over a length approximately equal to $\Delta \lambda_{p}$, where $\Delta \lambda_{p}$ is the difference in plasma 


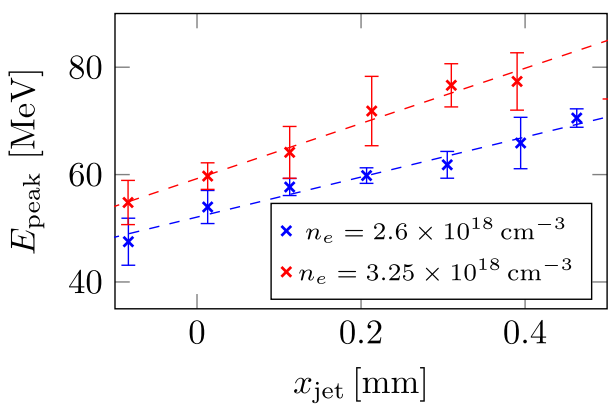

FIG. 3. Peak energy against relative jet position along the optical axis for two different plateau densities. The acceleration length in the plateau after the density down-ramp is controlled by the position of the gas jet. Zero on the $\mathrm{x}$-axis corresponds to the position where the density down-ramp is approximately centered in the density distribution from the jet. Each data point corresponds to 10 consecutive shots and the error bars indicate one standard deviation in each direction. While the electron number density in the peak is kept constant at $11 \times 10^{18} \mathrm{~cm}^{-3}$, the peak energy increases linearly (dashed blue line) with the relative jet position.

wavelength in the peak and the plateau regions. As the plasma wavelength increases from $11 \mu \mathrm{m}$ in the peak where the electron number density is $11 \times 10^{18} \mathrm{~cm}^{-3}$ to $19 \mu \mathrm{m}$ for the electron number density in the plateau of $3.25 \times 10^{18} \mathrm{~cm}^{-3}$, the injected electrons will be distributed along $8 \mu \mathrm{m}$ in the first plasma period. Thus, the injected electrons are distributed over approximately $40 \%$ of the first plasma wave period, and the average electric field experienced by the injected electrons is lower than if they were all placed in the back of the first plasma wave period, which is the case for self-injection.

The influence of the electron number density in the plateau after the density down-ramp was studied while keeping the electron number density in the peak constant at $11 \times 10^{18} \mathrm{~cm}^{-3}$. The resulting kinetic energy of the accelerated electrons showed a strong dependence on this electron number density (see Fig. 4).

While varying the energy of the electrons, using either of the methods described above, the charge did not show significant variations compared to the standard deviation. We conclude that the energy of the electrons could be controlled independently of the amount of injected charge, by changing either the electron number density in the plateau or the length of the plateau. The amount of charge in the electron beams could be separately controlled, within a certain range, by varying the peak density while keeping the plateau density constant. No trend is observed in the electron energy spectra while varying the peak density, whereas the beam charge shows a clear dependence on the electron number density in the peak as shown in Fig. 5. Up to an electron number density of $10 \times 10^{18} \mathrm{~cm}^{-3}$, the charge increases linearly with electron number density in the peak. By increasing this density by only $40 \%$ (from

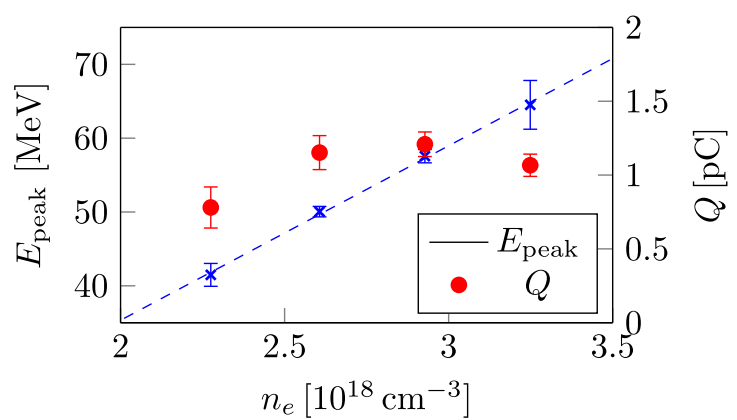

FIG. 4. Peak energy (blue) and total charge (red) against electron number density in the plateau. Each data point corresponds to 10 consecutive shots and the error bars indicate one standard deviation in each direction. The peak energy increases linearly (dashed blue line) with the electron number density, whereas the total charge shows no such trend. The electron number density in the peak is kept constant at $11 \times 10^{18} \mathrm{~cm}^{-3}$.

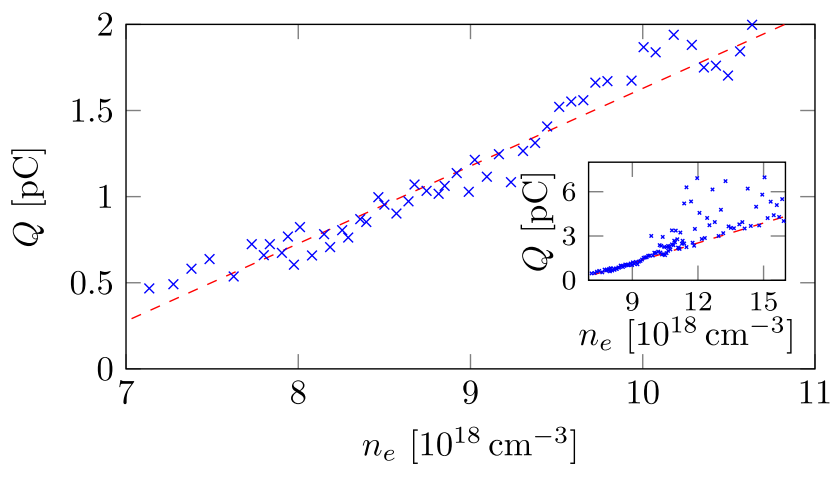

FIG. 5. Charge, above $40 \mathrm{MeV}$, as a function of electron number density in the peak. The positions of the two nozzles are kept fixed and the electron number density in the plateau is kept constant at $3.25 \times 10^{18} \mathrm{~cm}^{-3}$. At low peak densities, the amount of injected charge increases linearly with only small shotto-shot fluctuations (standard deviation of $0.09 \mathrm{pC}$ ) around the fitted line (red dashed). At densities above $10 \times 10^{18} \mathrm{~cm}^{-3}$ (shown in the inset), large fluctuations occur. However, the fluctuations only contribute to an increase in the total charge and indicate two different mechanisms of injection. The onset of the large fluctuations coincides with the electron number density threshold for self-injection.

$7.1 \times 10^{18} \mathrm{~cm}^{-3}$ to $10 \times 10^{18} \mathrm{~cm}^{-3}$ ), the observed charge was increased by more than a factor of 3 . Furthermore, the standard deviation of the shot-to-shot fluctuations in charge around the fitted linear dependence on peak electron number density is smaller than $0.1 \mathrm{pC}$ (standard deviation). Thus, the relative charge fluctuations are significantly smaller using this setup than in our experiments for selfinjection using a single gas jet.

An interesting feature is observed in the charge dependence as the electron number density in the peak is increased beyond $10 \times 10^{18} \mathrm{~cm}^{-3}$, shown in the inset in Fig. 5. At these densities the shot-to-shot fluctuations in charge are much larger than for lower densities. Remarkably, there 
is not a single data point below the line that follows the charge dependence for densities below $10 \times 10^{18} \mathrm{~cm}^{-3}$. The images of the dispersed electrons on the scintillating screen show that the electron beams, for peak densities above $10 \times 10^{18} \mathrm{~cm}^{-3}$ typically contain two components. One component with spectral shape and total charge similar to the ones observed at lower peak density is present on every shot. In addition, some beams contain a second component with higher charge and different spectral shape. The shot-to-shot fluctuations in this component is significantly larger than the fluctuations in the first component. We interpret this feature as injection of electrons through two different mechanisms; the stable, low charge component which is present on every shot is injected as the laser pulse propagate through the density down-ramp. The second component, which is only present above a certain threshold value for the electron number density in the peak, could be due to self-injection in the peak. This interpretation is supported by the observation that the value of the electron number density above which the second component starts to appear is the same as the electron number density threshold for self-injection observed in our experiments using a single gas jet.

To further support our interpretations of the experimental results, particle-in-cell simulations are performed using the code CALDER-CIRC [19]. In the simulations, the electron number density profile is approximated by a piecewise linear function, including two regions of constant density joined together by a linear gradient as shown in Fig. 6(a). The laser pulse parameters are chosen to correspond to those used in the experiments.

From the simulations, it is observed that the laser pulse undergoes self-focusing and self-compression in the increasing density and excite a highly nonlinear wakefield as the laser pulse reaches the density peak. However, no electrons are injected into the accelerating structure in this region [see Fig. 6(b)], as the wakefield is not yet strong enough for self-injection. As the laser pulse propagates through the linear density down-ramp, the wakefield structure increases in size and a certain portion of the background electrons become located within the electron void behind the laser pulse [see Fig. 6(c)]. The injection of electrons into the wakefield stops when the rear end of the first plasma period reaches the end of the density downramp, whereas the already injected electrons become further accelerated in the remaining plasma.

The observations from the simulations of injection in the density down-ramp and consecutive acceleration in the following plateau agree perfectly with the experimental observations presented above. For example, from Fig. 6(a) it is clear that the final energy of the electrons can be controlled by varying the length of the plasma after the density down-ramp.

It is further evident from the solid curves in Fig. 6(b-c) that the maximum accelerating field is much lower in the
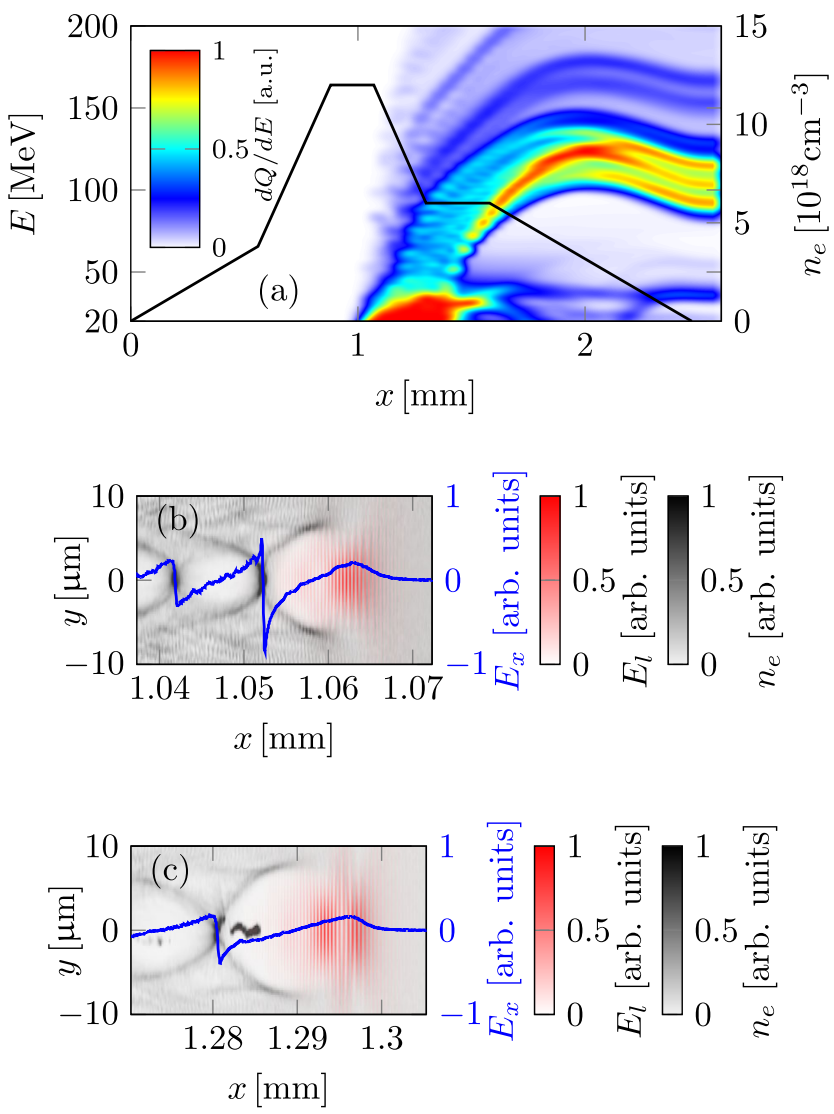

FIG. 6. Simulated evolution of the electron spectrum as the laser pulse propagates through the plasma (a) and local electron number density distribution before (b) and after (c) the density down-ramp along with the laser field (red) and accelerating electric field (blue). Injection of electrons into the wakefield structure occurs in the density down-ramp, located between $\approx 1.1 \mathrm{~mm}$ and $\approx 1.35 \mathrm{~mm}$. The injected electrons are accelerated in the density plateau and the final electron energy spectrum contains a peak centered around $105 \mathrm{MeV}$ and a FWHM of $20 \mathrm{MeV}$. In (c) electrons have been trapped after being injected as the plasma wavelength gradually increased in the density downramp. Thus, the electrons are distributed longitudinally over a distance approximately equal to $\Delta \lambda_{p} \approx 5 \mu \mathrm{m}$.

low electron number density of the plateau than in the peak. Also, since the electrons are distributed longitudinally the average electric field experienced by the injected electrons is lower than if all electrons would be located at the back of the first plasma wave period.

Furthermore, it is observed in the simulations that the wakefield structure is close to breaking already before the density down-ramp and only minor changes in the parameters for the simulation result in self-injection there. This agrees well with the experimental findings, in which two populations of accelerated electrons are identified at high densities (see Fig. 5).

The results from the simulations can also be used to understand the shape of electron energy spectra of the beams of accelerated electrons. In Fig. 7, the longitudinal 


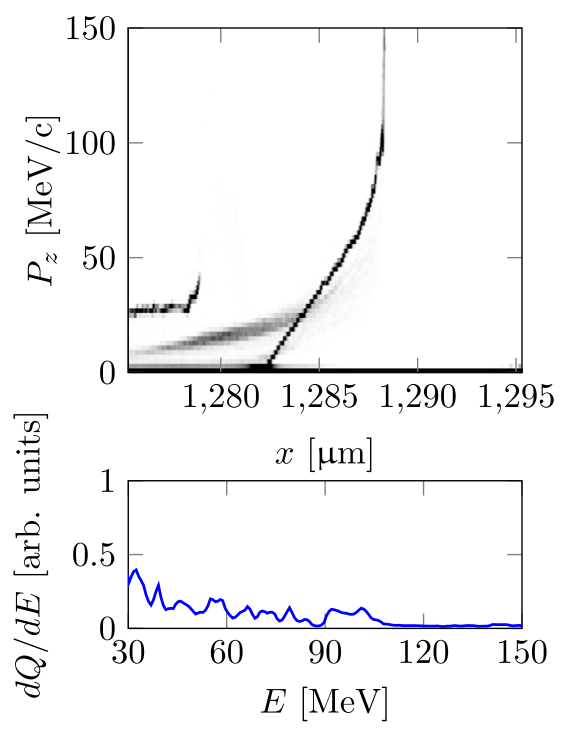

(a)

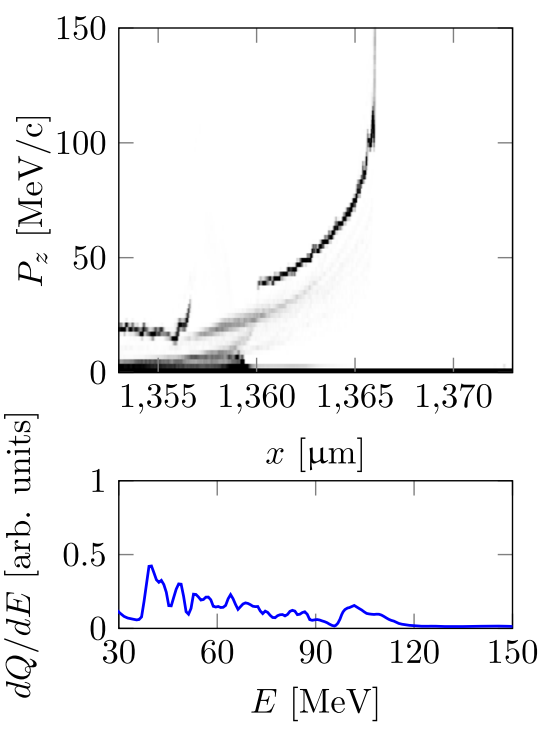

(b)

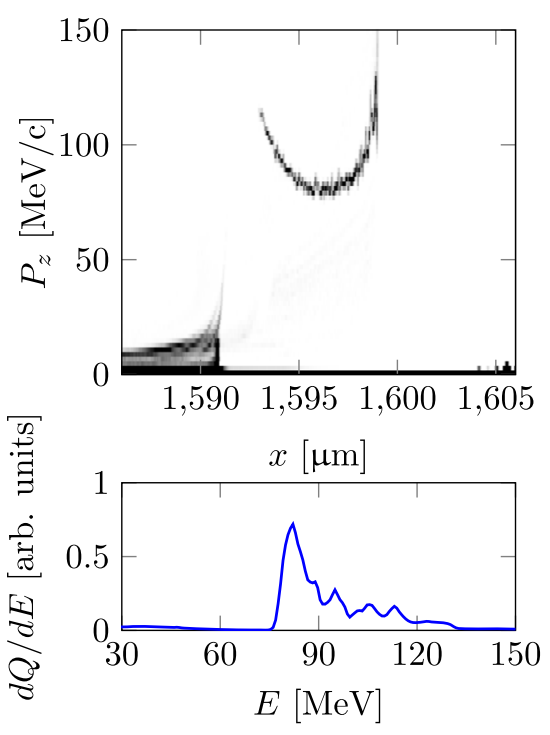

(c)

FIG. 7. Simulated compression of longitudinal phase-space distribution of injected electrons in the density plateau. In the first step (a) the laser pulse has just propagated through the density down-ramp, and the injected electrons are distributed along a line with a slope approximately equal to $12 \mathrm{MeV} / \mathrm{c} / \mu \mathrm{m}$, corresponding to a large energy spread in the total energy spectrum. After acceleration over $75 \mu \mathrm{m}$ (b), the average energy of the injected electrons has increased, and the phase-space distribution starts to diverge from a line. After further acceleration over $240 \mu \mathrm{m}$ (c), the phase-space distribution has deformed into a U-shape, corresponding to a peaked energy spectrum. The compression of the phase-space distribution to yield a peaked energy spectrum occurs because the injected electrons are distributed longitudinally, and thus experience different accelerating field. The slope of the front part of the injected electrons is too large, and this part of the electron distribution is not compressed.

phase-space distribution of the electrons is shown at three different locations along the optical axis following the laser pulse. Immediately after the density down-ramp [Fig. 7(a)], the injected electrons are distributed along a line in phasespace, corresponding to a spread both in energy and longitudinal position. After propagating a short distance, the average energy of the injected electrons is increased [Fig. 7(b)]. Furthermore, since the electrons have a longitudinal spread, they experience different electric field strength. Locally, this results in a rotation of the phasespace distribution of electrons, and globally the phasespace distribution appears to be bending. In the final step [Fig. 7(c)], the distribution of electrons has been deformed into a U-shape, corresponding to an increased distribution of electrons at an energy corresponding to the bottom of the U-shape. Thus, the rotation and bending of the original phase-space distribution of electrons thus acts to compress the energy spectrum.

In conclusion, we have demonstrated independent control of the number of injected electrons and their final energy distribution in a laser wakefield accelerator using a simple setup to tailor the density distribution. It has been shown that electrons are injected in the density down-ramp between two regions of different electron number density. The shot-to-shot fluctuations in both charge and energy are greatly improved in comparison to electron beams generated by self-injection. The same setup will be used in future experiments to study acceleration over longer distances and also to study localized ionization-induced injection.

\section{ACKNOWLEDGMENTS}

We acknowledge the support of the Swedish Research Council, the Knut and Alice Wallenberg Foundation, the Swedish Foundation for Strategic Research, LaserlabEurope/CHARPAC (Grant Agreement No. 284464, EC's 7th Framework Programme) and EuCARD2/ANAC2 (Grant Agreement No. 312453, EC's 7th Framework Programme).

[1] T. Tajima and J. M. Dawson, Phys. Rev. Lett. 43, 267 (1979).

[2] C. G. R. Geddes, C. Toth, J. van Tilborg, E. Esarey, C. B. Schroeder, D. Bruhwiler, C. Nieter, J. Cary, and W. P. Leemans, Nature (London) 431, 538 (2004).

[3] J. Faure, Y. Glinec, A. Pukhov, S. Kiselev, S. Gordienko, E. Lefebvre, J.-P. Rousseau, F. Burgy, and V. Malka, Nature (London) 431, 541 (2004).

[4] S. P. D. Mangles, C. D. Murphy, Z. Najmudin, A. G. R. Thomas, J. L. Collier, A. E. Dangor, E. J. Divall, P. S. Foster, J. G. Gallacher, C. J. Hooker, D. A. Jaroszynski, A. J. Langley, W. B. Mori, P. A. Norreys, F. S. Tsung, 
R. Viskup, B. R. Walton, and K. Krushelnick, Nature (London) 431, 535 (2004).

[5] D. Umstadter, J. K. Kim, and E. Dodd, Phys. Rev. Lett. 76, 2073 (1996).

[6] E. Esarey, R. F. Hubbard, W. P. Leemans, A. Ting, and P. Sprangle, Phys. Rev. Lett. 79, 2682 (1997).

[7] J. Faure, C. Rechatin, A. Norlin, A. Lifschitz, Y. Glinec, and V. Malka, Nature (London) 444, 737 (2006).

[8] A. Pak, K. A. Marsh, S. F. Martins, W. Lu, W. B. Mori, and C. Joshi, Phys. Rev. Lett. 104, 025003 (2010).

[9] C. McGuffey, A. G. R. Thomas, W. Schumaker, T. Matsuoka, V. Chvykov, F. J. Dollar, G. Kalintchenko, V. Yanovsky, A. Maksimchuk, K. Krushelnick, V. Y. Bychenkov, I. V. Glazyrin, and A. V. Karpeev, Phys. Rev. Lett. 104, 025004 (2010).

[10] M. Chen, E. Esarey, C. B. Schroeder, C. G. R. Geddes, and W. P. Leemans, Phys. Plasmas 19, 033101 (2012).

[11] F. G. Desforges, B. S. Paradkar, M. Hansson, J. Ju, L. Senje, T. L. Audet, A. Persson, S. Dobosz-Dufrénoy, O. Lundh, G. Maynard, P. Monot, J.-L. Vay, C.-G. Wahlström, and B. Cros, Phys. Plasmas 21, 120703 (2014).

[12] S. Bulanov, N. Naumova, F. Pegoraro, and J. Sakai, Phys. Rev. E 58, R5257 (1998).

[13] H. Suk, N. Barov, J. B. Rosenzweig, and E. Esarey, Phys. Rev. Lett. 86, 1011 (2001).

[14] A. J. Gonsalves, K. Nakamura, C. Lin, D. Panasenko, S. Shiraishi, T. Sokollik, C. Benedetti, C. B. Schroeder, C. G. R. Geddes, J. van Tilborg, J. Osterhoff, E. Esarey, C. Toth, and W. P. Leemans, Nat. Phys. 7, 862 (2011).

[15] M. Burza, A. Gonoskov, K. Svensson, F. Wojda, A. Persson, M. Hansson, G. Genoud, M. Marklund,
C.-G. Wahlström, and O. Lundh, Phys. Rev. ST Accel. Beams 16, 011301 (2013).

[16] G. Golovin, S. Chen, N. Powers, C. Liu, S. Banerjee, J. Zhang, M. Zeng, Z. Sheng, and D. Umstadter, Phys. Rev. ST Accel. Beams 18, 011301 (2015).

[17] J. Faure, C. Rechatin, O. Lundh, L. Ammoura, and V. Malka, Phys. Plasmas 17, 083107 (2010).

[18] K. Schmid, A. Buck, C. M. S. Sears, J. M. Mikhailova, R. Tautz, D. Herrmann, M. Geissler, F. Krausz, and L. Veisz, Phys. Rev. ST Accel. Beams 13, 091301 (2010).

[19] A. Lifschitz, X. Davoine, E. Lefebvre, J. Faure, C. Rechatin, and V. Malka, J. Comput. Phys. 228, 1803 (2009).

[20] G. R. Plateau, N. H. Matlis, C. G. R. Geddes, A. J. Gonsalves, S. Shiraishi, C. Lin, R. A. van Mourik, and W. P. Leemans, Rev. Sci. Instrum. 81, 033108 (2010).

[21] A. Buck, K. Zeil, A. Popp, K. Schmid, A. Jochmann, S. D. Kraft, B. Hidding, T. Kudyakov, C. M. S. Sears, L. Veisz, S. Karsch, J. Pawelke, R. Sauerbrey, T. Cowan, F. Krausz, and U. Schramm, Rev. Sci. Instrum. 81, 033301 (2010).

[22] S. P. D. Mangles, A. G. R. Thomas, O. Lundh, F. Lindau, M. C. Kaluza, A. Persson, C.-G. Wahlström, K. Krushelnick, and Z. Najmudin, Phys. Plasmas 14, 056702 (2007).

[23] M. Hansson, L. Senje, A. Persson, O. Lundh, C.-G. Wahlström, F. G. Desforges, J. Ju, T. L. Audet, B. Cros, S. D. Dufrénoy, and P. Monot, Phys. Rev. ST Accel. Beams 17, 031303 (2014).

[24] E. Esarey, C. B. Schroeder, and W. P. Leemans, Rev. Mod. Phys. 81, 1229 (2009). 